\title{
Fock majorization in bosonic quantum channels with a passive environment
}

\author{
Michael G Jabbour(D and Nicolas J Cerf \\ Quantum Information and Communication, École polytechnique de Bruxelles, CP \\ 165, Université libre de Bruxelles, 1050 Bruxelles, Belgium \\ E-mail: mjabbour@ulb.ac.be
}

Received 4 October 2018, revised 8 November 2018

Accepted for publication 14 November 2018

Published 8 February 2019

\begin{abstract}
We introduce a class of quantum channels called passive-environment bosonic channels. These channels are relevant from a quantum thermodynamical viewpoint because they correspond to the energy-preserving linear coupling of a bosonic system with a bosonic environment that is in a passive state (no energy can be extracted from it with a unitary transformation) followed by discarding the environment. The Fock-majorization relation defined in Jabbour et al (2016 New J. Phys. 18 073047) happens to be particularly useful in this context as, unlike regular majorization, it connects the disorder of a state together with its energy. Our main result here is the preservation of Fockmajorization across all passive-environment bosonic channels. This implies a similar preservation for regular majorization over the set of passive states, and also extends to the passive-environment bosonic channels whose Stinespring dilation involves an active Gaussian unitary. Beyond bosonic systems, the class of passive-environment operations as defined here naturally generalizes the set of thermal operations and is expected to provide new insights into the thermodynamics of quantum systems.
\end{abstract}

Keywords: passive-environment bosonic channels, Fock-majorization, passive states, majorization

(Some figures may appear in colour only in the online journal)

\section{Introduction}

Quantum thermodynamics has become a very active research area in recent years, aiming at a better understanding of thermal operations on individual quantum systems at the microscopic scale, see e.g. [1-4]. Among the objectives that are pursued, finding conditions to discriminate the permitted operations from the forbidden ones is of key importance, with a milestone in this direction being the recently uncovered existence of several second laws of thermodynamics 
[5]. In this context, majorization theory [6] has proven to be a powerful tool as it allows one to compare states in terms of disorder, which is a primordial concept when studying thermal operations on quantum systems (see, e.g. the notion of thermo-majorization [2]). Although most works in quantum thermodynamics have considered discrete (often finite-dimensional) quantum systems, we turn here to continuous-variable bosonic (infinite-dimensional) quantum systems.

The application of majorization relations to express conditions on the interconvertibility between quantum entangled states [7] has been successfully extended to probe the interconvertibility between Gaussian bosonic entangled states [8]. More fundamentally, Gaussian bosonic channels have been shown to respect several majorization relations. For example, the output state of a quantum-limited phase-insensitive (amplifier or loss) channel was proven to obey an infinite ladder of majorization relations when the input state is a Fock state [9, 10]. Beyond that, it was proven that the output state corresponding to the vacuum at the input of a phase-insensitive Gaussian bosonic channel majorizes every other output state [11], while the multimode extension of this relation was shown to hold too [12]. The key role of majorization in Gaussian bosonic channels is even more apparent from the property that, within the set of input states with a same spectrum, the passive state produces an output state that majorizes every other output state [13].

Here, we go a step further and exploit the tools based on majorization theory for bosonic channels in a thermodynamical context. The evolution of a quantum thermodynamical system can indeed be viewed as a completely-positive trace-preserving map applied to the system, that is, a quantum channel. We focus in this paper on bosonic quantum channels that are Gaussian-dilatable [14] (i.e. a Gaussian unitary can be used in the Stinespring dilation of the channel) and involve a passive environment (i.e. no energy can be extracted by applying a unitary operation on the environment). Interestingly, these so-called passive-environment bosonic channels encompass Gaussian channels but also a large class of non-Gaussian channels.

We address the question of whether a majorization relation is transferred across these channels viewed as a thermodynamical operation. Our results build on the notion of Fockmajorization (or energy-majorization) [15], and imply that any two input states that obey a Fock-majorization relation are transformed into output states obeying a similar relation. This majorization preservation property thus holds for a large class of thermodynamically relevant channels, going beyond the special case of Gaussian bosonic channels as considered in [15].

In section 2, we summarize the notion of passive states and their role in quantum thermodynamics. We then define the class of bosonic quantum channels with a passive environment, which is a natural generalization of the noisy operations and thermal operations used for modeling the dynamics of quantum thermodynamical systems. In section 3 , we briefly review the Fock-majorization relation as defined in [15] and compare it with regular majorization and thermo-majorization. In section 4, we prove the preservation of Fock-majorization relations across bosonic channels characterized by a passive Gaussian unitary and a passive environment, and then discuss the implication for regular majorization preservation over the set of passive states. A main ingredient of our proofs derives from the analysis of the generating function of the matrix elements of Gaussian unitaries in the Fock basis, which yields useful recurrence equations on these non-Gaussian objects [16]. In section 5, we extend these results to passive-environment channels in which the Gaussian unitary is active. Finally, in section 6, we give our conclusions. 


\section{Passive states and passive-environment bosonic channels}

Passive states are interesting when studying quantum systems from a thermodynamical point of view. They are defined as those quantum states from which no work can be extracted under Hamiltonian processes, making them the most stable states among all states that are reachable through a unitary transformation [17]. As a result, a passive state, denoted as $\rho^{\downarrow}$, is diagonal in the eigenbasis of the Hamiltonian of the system and is characterized by non-increasing eigenvalues when the energy of the corresponding eigenvectors increases. Mathematically speaking, it can be written as

$$
\rho^{\downarrow}=\sum_{i} \lambda_{i}^{\downarrow}\left|e_{i}\right\rangle\left\langle e_{i}\right| \quad \text { with } \lambda_{i+1}^{\downarrow} \leqslant \lambda_{i}^{\downarrow} \text { if } e_{i+1}>e_{i},
$$

where $\left|e_{i}\right\rangle$ are the eigenvectors and $e_{i}$ the corresponding eigenvalues of the Hamiltonian of the system. Interestingly, one can often 'activate' the work extraction from a passive state by jointly acting on it and an ancilla $[17,18]$. Suppose one has access to $n$ replicas of the passive state (in this example, the ancilla consists of $n-1$ replicas), then the joint system may not be passive anymore, allowing one to extract work by applying a unitary operation on the $n$ replicas. For a sufficiently large $n$, this is actually the case for almost all passive states except for thermal states. The latter are a special case of passive states whose eigenspectrum is given by a geometric distribution, which is characterized by a single parameter (e.g. the temperature). Remarkably, the tensor product of $n$ replicas of a thermal state remains passive and no work can be extracted from it (thermal states can be shown to be the only 'completely' passive states [17]). Furthermore, if one fixes the von Neumann entropy of a state, the thermal state is well-known to admit the lowest energy among all states (including the passive states) having this entropy [19]. Hence, we may categorize thermal states as the most stable states among all passive states having the same von Neumann entropy.

Passive states also arise in the context of modeling the dynamics of quantum thermodynamical systems, where some specific passive states are usually chosen as free 'resources'. When constructing a resource theory, one needs to define the set of permitted (free) state transformations [20]. This can be done by combining the following operations: composing the state with a fixed environment (viewed as a bath), acting on the resulting joint state with a unitary (usually chosen to conserve the energy), and finally discarding the environment. The environment is usually chosen to be thermal, which is a reasonable physical assumption. Still, one can also construct a simpler, less realistic model by choosing the maximally mixed state for the environment. By doing so, one obtains so-called noisy operations (NO), which have the form

$$
\mathcal{C}_{\mathrm{NO}}\left(\rho_{\mathrm{S}}\right)=\operatorname{Tr}_{\mathrm{E}}\left[U_{\mathrm{SE}}\left(\rho_{\mathrm{S}} \otimes \frac{\mathbb{I}_{\mathrm{E}}}{n_{\mathrm{E}}}\right) U_{\mathrm{SE}}^{\dagger}\right]
$$

where $\rho_{\mathrm{S}}$ is the state of the system, $\mathbb{I}_{\mathrm{E}}$ is the identity defined on the environment of dimension $n_{\mathrm{E}}$, and $U_{\mathrm{SE}}$ is an energy-conserving unitary acting on the system and the environment. When a state is transformed according to $\mathcal{C}_{\mathrm{NO}}$, the input can be shown to majorize the output for large enough $n_{\mathrm{E}}$ [21]. This can be intuitively understood by noticing that a state undergoing such a transformation gets more mixed (the maximally mixed state is indeed majorized by every other state). A more realistic model is obtained by choosing a thermal state $\tau_{\mathrm{E}}$ for the environment, resulting into the so-called thermal operations (TO),

$$
\mathcal{C}_{\mathrm{TO}}\left(\rho_{\mathrm{S}}\right)=\operatorname{Tr}_{\mathrm{E}}\left[U_{\mathrm{SE}}\left(\rho_{\mathrm{S}} \otimes \tau_{\mathrm{E}}\right) U_{\mathrm{SE}}^{\dagger}\right]
$$


A similar input-output relation can be proven in the case of thermal operations $\mathcal{C}_{\text {TO }}$, with majorization being replaced by thermo-majorization [2]. Roughly speaking, this corresponds to a modified majorization after rescaling the eigenvalues of the state with those of the thermal state. It means that the state of the system gets 'closer' to the thermal state of the environment (here, it is the thermal state that is is thermo-majorized by every other state).

In this paper, we introduce a class of quantum channels that generalizes equations (2) and (3), where the environment is chosen to be in any passive state (note that in $\mathcal{C}_{\mathrm{NO}}$ and $\mathcal{C}_{\mathrm{TO}}$, the environment is in a special case of a passive state). Since we focus on bosonic systems, we choose the environment to be passive in the eigenbasis of the Hamiltonian of the harmonic oscillator (i.e. the Fock basis), and fix the unitary $U_{\mathrm{SE}}$ to be a beam splitter (i.e. the realization of an energy-conserving linear coupling between bosonic systems). The result is a thermodynamical operation that we call a passive-environment bosonic channel (or simply passive channel), which is of the form

$$
\mathcal{B}_{\eta}^{\downarrow}\left(\rho_{\mathrm{S}}\right)=\operatorname{Tr}_{\mathrm{E}}\left[U_{\eta}^{\mathrm{BS}}\left(\rho_{\mathrm{S}} \otimes \sigma_{\mathrm{E}}^{\downarrow}\right) U_{\eta}^{\mathrm{BS} \dagger}\right],
$$

where $\sigma_{\mathrm{E}}^{\downarrow}=\sum_{i=0}^{\infty} \lambda_{i}^{\downarrow}|i\rangle\langle i|$ is the passive state of the environment, with $\lambda_{i+1}^{\downarrow} \leqslant \lambda_{i}^{\downarrow}$ and $|i\rangle$ denoting Fock states. The unitary $U_{\eta}^{\mathrm{BS}}$ corresponds to a beam splitter (BS) of transmittance $\eta$ (hence the symbol $\mathcal{B}$ for the channel). It couples the system mode $S$ with the passive environment mode $\mathrm{E}$ (hence the arrow in the notation $\mathcal{B}^{\downarrow}$ ) through the relation $\hat{a}_{\mathrm{S}} \rightarrow \sqrt{\eta} \hat{a}_{\mathrm{S}}+\sqrt{1-\eta} \hat{a}_{\mathrm{E}}$, where $\hat{a}_{\mathrm{S}}$ are $\hat{a}_{\mathrm{E}}$ are the bosonic mode operators for the system and environment, respectively [22].

Note that in contrast with $\mathcal{C}_{\mathrm{TO}}$, which corresponds to a Gaussian channel in the case of bosonic systems, the map $\mathcal{B}_{\eta}^{\downarrow}$ effects a non-Gaussian channel since the system is coupled (via a Gaussian unitary) to an environment state that is generally non-Gaussian (this is called a Gaussian-dilatable channel [14] since there exists a Stinespring dilation of the channel admitting a Gaussian unitary). In this sense, our study of majorization preservation for the map $\mathcal{B}_{\eta}^{\downarrow}$ in section 4 generalizes an earlier study of majorization preservation in Gaussian channels [15]. To be more general, we also consider in section 5 the class of Gaussian-dilatable channels with an active Gaussian unitary, namely a two-mode squeezer (TMS), the environment being again passive. These maps are noted $\mathcal{A}_{\mathrm{G}}^{\downarrow}$, where $G$ is the amplification gain, and the corresponding two-mode squeezer unitary $U_{\lambda}^{\mathrm{TMS}}$ with parameter $\lambda=(G-1) / G$ couples the system mode $\mathrm{S}$ with the passive environment mode $\mathrm{E}$ through the relation $\hat{a}_{\mathrm{S}} \rightarrow \sqrt{G} \hat{a}_{\mathrm{S}}+\sqrt{G-1} \hat{a}_{\mathrm{E}}^{\dagger}$ [22]. It turns out that $\mathcal{A}_{\mathrm{G}}^{\downarrow}$ exhibits similar properties as those of $\mathcal{B}_{\eta}^{\downarrow}$ in terms of Fock-majorization.

\section{Fock-majorization relation}

Before turning to the Fock-majorization relation, let us summarize the basics of the theory of majorization applied to quantum systems. Majorization provides a pre-order relation on quantum states, allowing us to compare them in terms of disorder. We say that a state $\rho$ majorizes another state $\sigma$, denoted as $\rho \succ \sigma$, when

$$
\sum_{i=1}^{n} r_{i}^{\downarrow} \geqslant \sum_{i=1}^{n} s_{i}^{\downarrow}, \quad \forall n \geqslant 1,
$$

where $\boldsymbol{r}^{\downarrow}\left(\boldsymbol{s}^{\downarrow}\right)$ is the vector of eigenvalues of $\rho(\sigma)$ arranged in non-increasing order. Whenever equation (5) is verified, it means that $\boldsymbol{s}=\mathrm{D} \boldsymbol{r}$, where $\boldsymbol{r}(\boldsymbol{s})$ is the vector of eigenvalues of $\rho(\sigma)$ and $\mathrm{D}$ is a bistochastic matrix [6], so that state $\sigma$ can be obtained from state $\rho$ by applying a 
random mixture of unitaries (hence, $\sigma$ is more disordered than $\rho$ ). In addition, $\rho \succ \sigma$ is equivalent to condition $\operatorname{Tr} f(\rho) \geqslant \operatorname{Tr} f(\sigma)$ for any convex function $f: \mathbb{R} \rightarrow \mathbb{R}$, which introduces a structure in terms of convex functions [6].

The concept of Fock-majorization was introduced in [15] and can more generally be viewed as energy-majorization when the Hamiltonian is not the one of the harmonic oscillator. For a harmonic oscillator (or a bosonic mode), we say that a state $\rho$ Fock-majorizes another state $\sigma$, denoted as $\rho \succ_{\mathrm{F}} \sigma$, when

$$
\operatorname{Tr}\left(P_{n} \rho\right) \geqslant \operatorname{Tr}\left(P_{n} \sigma\right), \quad \forall n \geqslant 0,
$$

where $P_{n}=\sum_{i=0}^{n}|i\rangle\langle i|$ is a projector onto the space spanned by the $n+1$ first Fock states $|i\rangle$ (which are the eigenstates of the Hamiltonian of the harmonic oscillator). This (pre)order relation only depends on the vectors of diagonal elements of $\rho$ and $\sigma$ in the eigenbasis of the Hamiltonian, i.e. the Fock basis. In contrast with regular majorization, these vectors are not ordered by decreasing values, but by increasing photon number ${ }^{1}$.

Fock-majorization bears some similarity with the relation called 'upper-triangular majorization' introduced in [23]. There, it was shown that two states obeying such a relation can be related by a so-called 'cooling' map, which happens to be a special case of the thermal operations (3) when the environment is set to zero temperature (it is in the vacuum state). Instead, Fock-majorization corresponds to a 'lower-triangular majorization' and can therefore be interpreted as a relation indicating the existence of a 'heating' (or 'amplifying') map between the two states [24]. Fock-majorization and upper-triangular majorization are both linked with thermo-majorization [2] as all these relations rely on the diagonal elements of the states in the energy eigenbasis (here, the Fock basis). Thermo-majorization can be viewed as a majorization relative to the thermal distribution ${ }^{2}$. In the case of bosonic systems, it amounts to arrange the scaled probabilities $p_{i} / \tau_{i}$ (instead of the probabilities $p_{i}$ ) by non-increasing order, where $p_{i}$ $\left(\tau_{i}\right)$ are the diagonal elements of state $\rho$ (of the thermal state). Doing the same reordering for the diagonal elements $q_{i}$ of state $\sigma$, thermo-majorization then requires verifying the standard condition on the cumulative probabilities $\sum_{i=1}^{n} p_{i} \geqslant \sum_{i=1}^{n} q_{i}, \forall n \geqslant 1$.

Finally, coming back to Fock-majorization, let us stress an interesting connection with energy. Relation (6) is equivalent to check that, for any $E$, the probability that the outcome of an energy measurement on $\rho$ is at most $E$ is greater than the probability that the outcome of an energy measurement on $\sigma$ is at most $E$. As shown in [15], this has a simple consequence in terms of mean energy. If $\rho$ Fock-majorizes $\sigma$, then the mean energy of $\sigma$ is greater than or equal to the one of $\rho$, that is, $\rho \succ_{\mathrm{F}} \sigma \Rightarrow \operatorname{Tr}(H \rho) \leqslant \operatorname{Tr}(H \sigma)$, where $H$ is the Hamiltonian of the harmonic oscillator. This property can actually be generalized to any increasing function of $H$, which turns the condition into an equivalence [24].

\section{Fock-majorization preservation in passive-environment channels}

The notion of a majorization-preserving quantum channel was defined in [15]. A channel $\Phi$ is called majorization-preserving whenever it is such that if $\rho \succ \sigma$, then $\Phi[\rho] \succ \Phi[\sigma]$. The central result of [15] was that all (phase-insensitive and phase-conjugate) Gaussian bosonic channels $\Phi_{\mathrm{G}}$ are majorization-preserving over the set of passive states. That is, given two passive states $\rho^{\downarrow}$ and $\sigma^{\downarrow}$, if $\rho^{\downarrow} \succ \sigma^{\downarrow}$, then $\Phi_{\mathrm{G}}\left[\rho^{\downarrow}\right] \succ \Phi_{\mathrm{G}}\left[\sigma^{\downarrow}\right]$ for all $\Phi_{\mathrm{G}}$. The proof relied on

${ }^{1}$ Such a definition of energy-majorization without prior sorting makes sense because there exists a natural way of ordering the elements, here the energy. This type of majorization relation is named 'unordered' majorization in [6].

${ }^{2}$ The majorization relation relative to $d$ (denoted as $\succ_{d}$ ) is defined in [6], where $d$ is some probability vector. The relation $p \succ_{d} q$ means that $q$ is 'closer' than $p$ to the vector $d$. In regular majorization, $d$ is simply the uniform distribution, while in thermo-majorization it is the thermal distribution. 
the Fock-majorization relation and the fact that it coincides with regular majorization for passive states (i.e. $\rho^{\downarrow} \succ \sigma^{\downarrow} \Leftrightarrow \rho^{\downarrow} \succ_{\mathrm{F}} \sigma^{\downarrow}$ ). As a matter of fact, Gaussian channels $\Phi_{\mathrm{G}}$ were first proven to be Fock-majorization preserving, where a Fock-majorization preserving channel $\Phi$ is of course defined as a channel such that if $\rho \succ_{\mathrm{F}} \sigma$, then $\Phi[\rho] \succ_{\mathrm{F}} \Phi[\sigma]$. The preservation of Fock-majorization across channels $\Phi_{\mathrm{G}}$ was actually the key result of [15], from which the rest follows. It was proven based on the following theorem.

Theorem 1 ([15]). A channel $\Phi$ satisfying the condition $\langle n|\Phi[|i\rangle\langle j|]| n\rangle=0, \forall i \neq j, \forall n$ is Fock-majorization preserving if and only if it obeys the ladder of Fock-majorization relations

$$
\Phi[|i\rangle\langle i|] \succ_{\mathrm{F}} \Phi[|i+1\rangle\langle i+1|], \quad \forall i \geqslant 0 .
$$

In [15], all Gaussian channels $\Phi_{\mathrm{G}}$ were indeed shown to verify equation (7). Since they form a special case of passive-environment bosonic channels ${ }^{3}$, it is natural to investigate whether the Fock-majorization preservation property extends to all passive-environment bosonic channels $\mathcal{B}_{\eta}^{\downarrow}$ (and similarly $\mathcal{A}_{\mathrm{G}}^{\downarrow}$ ).

In order to prove this, we again recourse to theorem 1 , with a minor caveat. Indeed, the proof of theorem 1 in [15] did not mention the condition $\langle n|\Phi[|i\rangle\langle j|]| n\rangle=0, \forall i \neq j, \forall n$ since only Fock-diagonal states were considered at the input of channel $\Phi_{\mathrm{G}}$. However, theorem 1 also applies to input states that are non-diagonal in the Fock basis as long as the above condition is fulfilled (i.e. provided the non-diagonal elements of the input state do not contribute to the diagonal elements of the output state, which are the only ones that matter in the Fockmajorization relation) [24]. As shown in appendix A, this condition is verified for Gaussiandilatable channels with a passive environment, so theorem 1 can be applied to these channels. Before doing so, we are left with proving the following theorem.

Theorem 2. Passive-environment bosonic channels $\mathcal{B}_{\eta}^{\downarrow}$ exhibit the ladder of Fockmajorization relations

$$
\mathcal{B}_{\eta}^{\downarrow}[|i\rangle\langle i|] \succ_{\mathrm{F}} \mathcal{B}_{\eta}^{\downarrow}[|i+1\rangle\langle i+1|], \quad \forall i \geqslant 0 .
$$

Proof. We begin by proving the ladder of Fock-majorization relations for a passive channel $\mathcal{B}_{\eta}^{[K]}$ characterized by an environment that is a projector onto the space spanned by the $K+1$ first Fock states $|k\rangle$, i.e.

$$
\mathcal{B}_{\eta}^{[K]}(\rho)=\operatorname{Tr}_{\mathrm{E}}\left[U_{\eta}^{\mathrm{BS}}\left(\rho \otimes P_{K}^{\downarrow}\right) U_{\eta}^{\mathrm{BS} \dagger}\right],
$$

where $P_{K}^{\downarrow}=\sum_{k=0}^{K}|k\rangle\langle k|$. Note that $\mathcal{B}_{\eta}^{[K]}$ is not trace-preserving here since $P_{K}^{\downarrow}$ is not normalized. We need to show that $\mathcal{B}_{\eta}^{[K]}[|i\rangle\langle i|] \succ_{\mathrm{F}} \mathcal{B}_{\eta}^{[K]}[|i+1\rangle\langle i+1|], \forall i \geqslant 0$, or

$$
\operatorname{Tr}\left[P_{n}\left(\mathcal{B}_{\eta}^{[K]}[|i\rangle\langle i|]-\mathcal{B}_{\eta}^{[K]}[|i+1\rangle\langle i+1|]\right)\right] \geqslant 0, \quad \forall i \geqslant 0, \forall n \geqslant 0 .
$$

In [16], it was shown that if the environment is in a single Fock state $|k\rangle$, the action of the corresponding channel on a Fock state $|i\rangle$ can be written as

\footnotetext{
${ }^{3}$ In particular, the lossy Gaussian channels (i.e. channels whose Stinespring dilation gives a beam splitter) are passive-environment bosonic channels of the form (4), where the environment is chosen to be in a thermal (hence, passive) Gaussian state.
} 


$$
\operatorname{Tr}_{\mathrm{E}}\left[U_{\eta}^{\mathrm{BS}}(|i\rangle\langle i|\otimes| k\rangle\langle k|) U_{\eta}^{\mathrm{BS} \dagger}\right]=\sum_{m=0}^{i+k} B_{m}^{(i, k)}|m\rangle\langle m|
$$

where the coefficients $B_{m}^{(i, k)}$ obey the recurrence relation

$$
B_{m}^{(i, k)}=\eta B_{m-1}^{(i-1, k)}+(1-\eta) B_{m}^{(i-1, k)}+\eta B_{m}^{(i, k-1)}+(1-\eta) B_{m-1}^{(i, k-1)}-B_{m-1}^{(i-1, k-1)},
$$

when $i \geqslant 0, k \geqslant 0$ and $0 \leqslant m \leqslant i+k$. Whenever one of the indices $i, k, m$ is equal to zero in the left-hand side of equation (12), the coefficients with negative indices have to be removed on its right-hand side except if all indices are equal to zero, in which case the 'initial condition' is $B_{0}^{(0,0)}=1$. Using these notations, we need to prove that

$$
\Delta_{n}^{(i, K)}=\sum_{k=0}^{K} \sum_{m=0}^{n}\left[B_{m}^{(i, k)}-B_{m}^{(i+1, k)}\right] \geqslant 0, \quad \forall i \geqslant 0, \forall n \geqslant 0, n \leqslant i+k .
$$

Using the recurrence relation (12), we have that

$$
\begin{aligned}
\Delta_{n}^{(i, K)}= & \sum_{k=0}^{K} \sum_{m=0}^{n}\left[B_{m}^{(i, k)}-(1-\eta) B_{m}^{(i, k)}\right] \\
& -\sum_{k=0}^{K} \sum_{m=0}^{n}\left[\eta B_{m-1}^{(i, k)}+(1-\eta) B_{m-1}^{(i+1, k-1)}+\eta B_{m}^{(i+1, k-1)}-B_{m-1}^{(i, k-1)}\right] \\
= & \eta \sum_{k=0}^{K} \sum_{m=0}^{n}\left(B_{m}^{(i, k)}-B_{m-1}^{(i, k)}\right)-\eta \sum_{k=0}^{K} \sum_{m=0}^{n}\left(B_{m}^{(i+1, k-1)}-B_{m-1}^{(i+1, k-1)}\right) \\
& +\sum_{k=0}^{K} \sum_{m=0}^{n}\left(B_{m-1}^{(i, k-1)}-B_{m-1}^{(i+1, k-1)}\right) \\
= & \eta \sum_{k=0}^{K} B_{n}^{(i, k)}-\eta \sum_{k=0}^{K} B_{n}^{(i+1, k-1)}+\sum_{k=0}^{K-1} \sum_{m=0}^{n-1}\left(B_{m}^{(i, k)}-B_{m}^{(i+1, k)}\right) \\
= & \eta \sum_{k=0}^{K-1} B_{n}^{(i, k)}+\eta B_{n}^{(i, K)}-\eta \sum_{k=0}^{K-1} B_{n}^{(i+1, k)} \\
& +\eta \sum_{k=0}^{K-1} \sum_{m=0}^{n-1}\left(B_{m}^{(i, k)}-B_{m}^{(i+1, k)}\right)+(1-\eta) \Delta_{n-1}^{(i, K-1)} \\
= & \eta B_{n}^{(i, K)}+\eta \Delta_{n}^{(i, K-1)}+(1-\eta) \Delta_{n-1}^{(i, K-1)}
\end{aligned}
$$

For $K=0$, we know that $\Delta_{n}^{(i, 0)} \geqslant 0, \forall i \geqslant 0, \forall n \geqslant 0$ since $\mathcal{B}_{\eta}^{[0]}$ corresponds to a Gaussian pure-loss channel [15]. We are then able to prove equation (13) by using a recursion on $K$, since $B_{n}^{(i, k)} \geqslant 0, \forall i \geqslant 0, \forall n \geqslant 0, \forall k \geqslant 0$. This implies that 


$$
\mathcal{B}_{\eta}^{[K]}[|i\rangle\langle i|] \succ_{\mathrm{F}} \mathcal{B}_{\eta}^{[K]}[|i+1\rangle\langle i+1|], \quad \forall i \geqslant 0
$$

Now, since any passive state can be written as a convex sum over $K$ of (normalised) projectors $P_{K}^{\downarrow}$, the channels $\mathcal{B}_{\eta}^{\downarrow}$ can also be written as a convex combination of channels $\mathcal{B}_{\eta}^{[K]}$, hence we get the same Fock-majorization relation for channels $\mathcal{B}_{\eta}^{\downarrow}$, which concludes the proof of theorem 2 .

Using theorems 1 and 2, we obtain the following corollary.

Corollary 1. Passive-environment bosonic channels $\mathcal{B}_{\eta}^{\downarrow}$ are Fock-majorization preserving, that is, for all states $\rho$ and $\sigma$,

$$
\text { if } \rho \succ_{\mathrm{F}} \sigma \text {, then } \mathcal{B}_{\eta}^{\downarrow}[\rho] \succ_{\mathrm{F}} \mathcal{B}_{\eta}^{\downarrow}[\sigma] \text {. }
$$

Just like Gaussian channels, passive-environment bosonic channels do not preserve regular majorization, that is, if $\rho \succ \sigma$, then we cannot conclude that $\mathcal{B}_{\eta}^{\downarrow}[\rho] \succ \mathcal{B}_{\eta}^{\downarrow}[\sigma]$. Counterexamples can be easily found. However, one can prove that passive-environment bosonic channels become majorization preserving when restricting to the set of passive states. Because of the equivalence between majorization and Fock-majorization for this set, we simply need to verify that passive states remain passive after evolving through the channel. This is the content of the following theorem.

Theorem 3. Passive-environment bosonic channels $\mathcal{B}_{\eta}^{\downarrow}$ are passive preserving, that is

$$
\text { if } \rho^{\downarrow} \text { is passive, then } \mathcal{B}_{\eta}^{\downarrow}\left[\rho^{\downarrow}\right] \text { is also passive. }
$$

Proof. We begin by showing that this theorem is true for any passive channel $\mathcal{B}_{\eta}^{[K]}$, but when the input is the (unnormalized) projector $P_{I}^{\downarrow}$. We need to prove that

$$
\operatorname{Tr}\left[(|n\rangle\langle n|-| n+1\rangle\langle n+1|) \mathcal{B}_{\eta}^{[K]}\left[P_{I}^{\downarrow}\right]\right] \geqslant 0, \quad \forall I \geqslant 0, \forall n \geqslant 0,
$$

or,

$$
\Gamma_{n}^{(I, K)}=\sum_{i=0}^{I} \sum_{k=0}^{K}\left(B_{n}^{(i, k)}-B_{n+1}^{(i, k)}\right) \geqslant 0, \quad \forall I \geqslant 0, \forall n \geqslant 0 .
$$

Using the recurrence relation (12), we have that 


$$
\begin{aligned}
& \Gamma_{n}^{(I, K)}= \sum_{i=0}^{I} \sum_{k=0}^{K}\left(B_{n}^{(i, k)}-\eta B_{n}^{(i-1, k)}-(1-\eta) B_{n+1}^{(i-1, k)}-\eta B_{n+1}^{(i, k-1)}\right. \\
&\left.-(1-\eta) B_{n}^{(i, k-1)}+B_{n}^{(i-1, k-1)}\right) \\
&= \eta \sum_{i=0}^{I} \sum_{k=0}^{K}\left(B_{n}^{(i, k)}-B_{n}^{(i-1, k)}\right)+(1-\eta) \sum_{i=0}^{I} \sum_{k=0}^{K}\left(B_{n}^{(i, k)}-B_{n}^{(i, k-1)}\right) \\
&-(1-\eta) \sum_{i=0}^{I} \sum_{k=0}^{K} B_{n+1}^{(i-1, k)}-\eta \sum_{i=0}^{I} \sum_{k=0}^{K} B_{n+1}^{(i, k-1)}+\sum_{i=0}^{I} \sum_{k=0}^{K} B_{n}^{(i-1, k-1)} \\
&= \quad \sum_{k=0}^{K} B_{n}^{(i, k)}+(1-\eta) \sum_{i=0}^{I} B_{n}^{(i, k)}-(1-\eta) \sum_{i=0}^{I-1} \sum_{k=0}^{K} B_{n+1}^{(i, k)} \\
&-\eta \sum_{i=0}^{I} \sum_{k=0}^{K-1} B_{n+1}^{(i, k)}+\sum_{i=0}^{I-1} \sum_{k=0}^{K-1} B_{n}^{(i, k)} \\
&= B_{n}^{(i, k)}-(1-\eta) \sum_{i=0}^{I-1} \sum_{k=0}^{K} B_{n+1}^{(i, k)}-\eta \sum_{i=0}^{I} \sum_{k=0}^{K-1} B_{n+1}^{(i, k)}+\eta \sum_{i=0}^{I} \sum_{k=0}^{K-1} B_{n}^{(i, k)} \\
&+(1-\eta) \sum_{i=0}^{I-1} \sum_{k=0}^{K} B_{n}^{(i, k)} \\
&= B_{n}^{(i, k)}+\eta \sum_{i=0}^{I} \sum_{k=0}^{K-1}\left(B_{n}^{(i, k)}-B_{n+1}^{(i, k)}\right)+(1-\eta) \sum_{i=0}^{I-1} \sum_{k=0}^{K}\left(B_{n}^{(i, k)}-B_{n+1}^{(i, k)}\right) \\
&+\eta \Gamma_{n}^{(I, K-1)}+(1-\eta) \Gamma_{n}^{(I-1, K)} .
\end{aligned}
$$

We know that $\Gamma_{n}^{(I, 0)} \geqslant 0, \forall I \geqslant 0, \forall n \geqslant 0$, since it corresponds to a Gaussian pure-loss channel, and was proven in [15]. We also know, because of the symmetry of the beam splitter, that $\Gamma_{n}^{(0, K)} \geqslant 0, \forall K \geqslant 0, \forall n \geqslant 0$. We are then able to prove (19) by using a recursive argument on both $I$ and $K$, since $B_{n}^{(i, k)} \geqslant 0, \forall I \geqslant 0, \forall K \geqslant 0, \forall n \geqslant 0$. This shows that $\mathcal{B}_{\eta}^{[K]}\left[P_{I}^{\downarrow}\right]$ is passive. As before, we conclude the proof by using the fact that any passive state can be written as a convex sum of (normalised) projectors $P_{l}^{\downarrow}$.

Using corollary 1 and theorem 3, we are now able to state the following.

Corollary 2. Passive-environment bosonic channels $\mathcal{B}_{\eta}^{\downarrow}$ are majorization-preserving over the set of passive states, that is, for any two passive states $\rho^{\downarrow}$ and $\sigma^{\downarrow}$,

$$
\text { if } \rho^{\downarrow} \succ \sigma^{\downarrow} \text {, then } \mathcal{B}_{\eta}^{\downarrow}\left[\rho^{\downarrow}\right] \succ \mathcal{B}_{\eta}^{\downarrow}\left[\sigma^{\downarrow}\right] \text {. }
$$

\section{Passive-environment channels with an active Gaussian unitary}

For completeness, we now show that all the results of section 4 extend to the passive-environment channels obtained with an active Gaussian unitary, namely $\mathcal{A}_{\mathrm{G}}^{\downarrow}$. We will recourse to the following theorem. 
Theorem 4 ([15]). A channel $\Phi$ satisfying the condition $\langle n|\Phi[|i\rangle\langle i|]| m\rangle=0, \forall n \neq m, \forall i$ is passive preserving if and only if its adjoint channel $\phi^{\dagger}$ obeys the ladder of Fock-majorization relations

$$
\Phi^{\dagger}[|i\rangle\langle i|] \succ_{\mathrm{F}} \Phi^{\dagger}[|i+1\rangle\langle i+1|], \quad \forall i \geqslant 0 .
$$

Again, compared to the statement of theorem 4 in [15], we have added the condition that the diagonal elements at the input of the channel do not yield non-diagonal elements at the output of the channel [24]. This condition is fulfilled for Gaussian-dilatable channels with a passive environment, see appendix A.

In order to exploit theorem 4, the last thing that remains to be done is to prove the duality between channels $\mathcal{B}_{\eta}^{\downarrow}$ and $\mathcal{A}_{\mathrm{G}}^{\downarrow}$. For completeness, we will investigate the duality relation for Gaussian-dilatable channels involving arbitrary states of the environment in the next theorem. Then, we will focus on the special case of channels with Fock-diagonal environments, more precisely channels $\mathcal{B}_{\eta}^{\downarrow}$ built on a beam splitter of transmittance $\eta$ and channels $\mathcal{A}_{\mathrm{G}}^{\downarrow}$ based on a two-mode squeezer with a gain $G$. The corresponding unitaries can be expressed as

$$
\begin{aligned}
& U_{\eta}^{\mathrm{BS}}=\exp \left[\theta\left(\hat{a}_{\mathrm{S}}^{\dagger} \hat{a}_{\mathrm{E}}-\hat{a}_{\mathrm{S}} \hat{a}_{\mathrm{E}}^{\dagger}\right)\right], \quad \eta=\cos ^{2} \theta \in[0,1], \\
& U_{\lambda}^{\mathrm{TMS}}=\exp \left[\frac{r}{2}\left(\hat{a}_{\mathrm{S}} \hat{a}_{\mathrm{E}}-\hat{a}_{\mathrm{S}}^{\dagger} \hat{a}_{\mathrm{E}}^{\dagger}\right)\right], \quad \lambda=\tanh ^{2} r \in[0,1] .
\end{aligned}
$$

As it so happens, when the environment is not diagonal in the Fock basis, one needs to characterize the channel using parameters $\theta$ and $r$ of equations (23) and (24), as a phase may be introduced depending on their signs. In order to do so, we relabel the above two unitaries as

$$
\tilde{U}_{\theta}^{\mathrm{BS}}=\exp \left[\theta\left(\hat{a}_{\mathrm{S}}^{\dagger} \hat{a}_{\mathrm{E}}-\hat{a}_{\mathrm{S}} \hat{a}_{\mathrm{E}}^{\dagger}\right)\right], \quad \tilde{U}_{r}^{\mathrm{TMS}}=\exp \left[\frac{r}{2}\left(\hat{a}_{\mathrm{S}} \hat{a}_{\mathrm{E}}-\hat{a}_{\mathrm{S}}^{\dagger} \hat{a}_{\mathrm{E}}^{\dagger}\right)\right] .
$$

We prove the following duality theorem for Gausian-dilatable channels involving arbitrary environments.

Theorem 5. Consider a channel $\tilde{\mathcal{C}}_{\theta}^{\mathrm{BS}}$ whose Stinespring dilation is based on a beam splitter of parameter $\theta$, i.e.

$$
\tilde{\mathcal{C}}_{\theta}^{\mathrm{BS}}[\bullet]=\operatorname{Tr}_{\mathrm{E}}\left[\tilde{U}_{\theta}^{\mathrm{BS}}\left(\bullet \otimes \sigma_{\mathrm{E}}^{(1)}\right) \tilde{U}_{\theta}^{\mathrm{BS} \dagger}\right],
$$

and a channel $\tilde{\mathcal{C}}_{r}^{\text {TMS }}$ having a two-mode squeezer of parameter $r$ in its Stinespring dilation, i.e.

$$
\tilde{\mathcal{C}}_{r}^{\mathrm{TMS}}[\bullet]=\operatorname{Tr}_{\mathrm{E}}\left[\tilde{U}_{r}^{\mathrm{TMS}}\left(\bullet \otimes \sigma_{\mathrm{E}}^{(2)}\right) \tilde{U}_{r}^{\mathrm{TMS} \dagger}\right] .
$$

The adjoint map of $\tilde{\mathcal{C}}_{\theta}^{\mathrm{BS}}$ is given by

$$
\left(\tilde{\mathcal{C}}_{\theta}^{\mathrm{BS}}\right)^{\dagger}=\frac{1}{\cos ^{2} \theta} \tilde{\mathcal{C}}_{-r}^{\mathrm{TMS}},
$$

where $\theta$ and $r$ are related by $\cos \theta=1 / \cosh r$, and $\sigma_{\mathrm{E}}^{(2)}=\left(\sigma_{\mathrm{E}}^{(1)}\right)^{\mathrm{T}}$ with $T$ denoting matrix transposition in the Fock basis. 
Proof. We start with two states expressed in the Fock basis as

$$
\rho=\sum_{i, j} \rho_{i, j}|i\rangle\langle j|, \quad \text { and } \quad \gamma=\sum_{n, m} \gamma_{n, m}|n\rangle\langle m|,
$$

and compute the object

$$
\operatorname{Tr}\left[\gamma \tilde{\mathcal{C}}_{\theta}^{\mathrm{BS}}[\rho]\right]=\sum_{n, m} \gamma_{n, m} \sum_{i, j} \rho_{i, j}\left\langle m\left|\tilde{\mathcal{C}}_{\theta}^{\mathrm{BS}}[|i\rangle\langle j|]\right| n\right\rangle .
$$

If we consider a general environment $\sigma_{\mathrm{E}}^{(1)}=\sum_{k, l} \sigma_{k, l}^{(1)}|k\rangle\langle l|$, we get

$$
\operatorname{Tr}\left[\gamma \tilde{\mathcal{C}}_{\theta}^{\mathrm{BS}}[\rho]\right]=\sum_{n, m} \gamma_{n, m} \sum_{i, j} \rho_{i, j} \sum_{k, l} \sigma_{k, l}^{(1)} \sum_{e}\left\langle m, e\left|\tilde{U}_{\theta}^{\mathrm{BS}}\right| i, k\right\rangle\left\langle j, l\left|\tilde{U}_{\theta}^{\mathrm{BS} \dagger}\right| n, e\right\rangle .
$$

It was shown in [16] that, under partial time reversal, a beam splitter is turned into a two-mode squeezer, or, more precisely, their respective transition amplitudes in the Fock basis are related through

$$
\left\langle m, e\left|\tilde{U}_{\theta}^{\mathrm{BS}}\right| i, k\right\rangle=\frac{1}{\cos \theta}\left\langle m, k\left|\tilde{U}_{r}^{\mathrm{TMS}}\right| i, e\right\rangle
$$

where $\theta$ and $r$ verify $\cos \theta=1 / \cosh r$. Notice that the ket and bra of the second mode have been swapped in equation (32). This property leads to

$$
\begin{aligned}
\operatorname{Tr}\left[\gamma \tilde{\mathcal{C}}_{\theta}^{\mathrm{BS}}[\rho]\right] & =\frac{1}{\cos ^{2} \theta} \sum_{n, m} \gamma_{n, m} \sum_{i, j} \rho_{i, j} \sum_{k, l} \sigma_{k, l}^{(1)} \sum_{e}\left\langle m, k\left|\tilde{U}_{r}^{\mathrm{TMS}}\right| i, e\right\rangle\left\langle j, e\left|\tilde{U}_{r}^{\mathrm{TMS} \dagger}\right| n, l\right\rangle \\
& =\frac{1}{\cos ^{2} \theta} \sum_{n, m} \gamma_{n, m} \sum_{i, j} \rho_{i, j} \sum_{k, l} \sigma_{k, l}^{(1)} \sum_{e}\left\langle j, e\left|\tilde{U}_{r}^{\mathrm{TMS} \dagger}\right| n, l\right\rangle\left\langle m, k\left|\tilde{U}_{r}^{\mathrm{TMS}}\right| i, e\right\rangle .
\end{aligned}
$$

In view of equation (25), we have $\tilde{U}_{r}^{\mathrm{TMS} \dagger}=\tilde{U}_{-r}^{\mathrm{TMS}}$, so that

$\operatorname{Tr}\left[\gamma \tilde{\mathcal{C}}_{\theta}^{\mathrm{BS}}[\rho]\right]=\frac{1}{\cos ^{2} \theta} \sum_{n, m} \gamma_{n, m} \sum_{i, j} \rho_{i, j} \sum_{k, l} \sigma_{k, l}^{(1)} \sum_{e}\left\langle j, e\left|\tilde{U}_{-r}^{\mathrm{TMS}}\right| n, l\right\rangle\left\langle m, k\left|\tilde{U}_{-r}^{\mathrm{TMS} \dagger}\right| i, e\right\rangle$.

As a consequence,

$\operatorname{Tr}\left[\gamma \tilde{\mathcal{C}}_{\theta}^{\mathrm{BS}}[\rho]\right]=\frac{1}{\cos ^{2} \theta} \sum_{n, m} \gamma_{n, m} \sum_{i, j} \rho_{i, j}\left\langle m\left|\tilde{\mathcal{C}}_{-r}^{\mathrm{TMS}}[|i\rangle\langle j|]\right| n\right\rangle=\frac{1}{\cos ^{2} \theta} \operatorname{Tr}\left[\rho \tilde{\mathcal{C}}_{-r}^{\mathrm{TMS}}[\gamma]\right]$,

where the environment $\sigma_{\mathrm{E}}^{(2)}$ characterizing the channel $\tilde{\mathcal{C}}_{-r}^{\mathrm{TMS}}$ is related to the environment $\sigma_{\mathrm{E}}^{(1)}$ of $\tilde{\mathcal{C}}_{\theta}^{\mathrm{BS}}$ through $\sigma_{\mathrm{E}}^{(2)}=\left(\sigma_{\mathrm{E}}^{(1)}\right)^{\mathrm{T}}$. Thus, the adjoint of channel $\tilde{\mathcal{C}}_{\theta}^{\mathrm{BS}}$ verifies equation (28) where one should transpose the environment in the Fock basis.

When the environment which characterizes the channel is diagonal in the Fock basis, equation (28) can be simplified. Indeed, we show in appendix B that in this case, $\tilde{\mathcal{C}}_{-\theta}^{\mathrm{BS}}=\tilde{\mathcal{C}}_{\theta}^{\mathrm{BS}}$ and $\tilde{\mathcal{C}}_{-r}^{\mathrm{TMS}}=\tilde{\mathcal{C}}_{r}^{\mathrm{TMS}}$. This leads to the following corollary of theorem 5 . 
Corollary 3. Consider a channel

$$
\mathcal{C}_{\eta}^{\mathrm{BS}}[\bullet]=\operatorname{Tr}_{\mathrm{E}}\left[U_{\eta}^{\mathrm{BS}}\left(\bullet \otimes \sigma_{\mathrm{E}}\right) U_{\eta}^{\mathrm{BS} \dagger}\right]
$$

where the state of the environment $\sigma_{\mathrm{E}}$ is diagonal in the Fock basis. Its adjoint map $\left(\mathcal{C}_{\eta}^{\mathrm{BS}}\right)^{\dagger}$ is such that

$$
\left(\mathcal{C}_{\eta}^{\mathrm{BS}}\right)^{\dagger}=\frac{1}{\eta} \mathcal{C}_{1-\eta}^{\mathrm{TMS}}
$$

with

$$
\mathcal{C}_{\lambda}^{\mathrm{TMS}}[\bullet]=\operatorname{Tr}_{\mathrm{E}}\left[U_{\lambda}^{\mathrm{TMS}}\left(\bullet \otimes \sigma_{\mathrm{E}}\right) U_{\lambda}^{\mathrm{TMS} \dagger}\right]
$$

In a two-mode squeezer, the parameter $\lambda$ is related to the parametric gain $G$ via $\lambda=(G-1) / G$, so that the relation $\lambda=1-\eta$ translates into $G=1 / \eta$. Thus, in the special case of passive-environment channels (for which the transpose of the environment state remains unchanged) the adjoint map of $\mathcal{B}_{\eta}^{\downarrow}$ is $\frac{1}{\eta} \mathcal{A}_{1 / \eta}^{\downarrow}$, in full analogy with the situation for Gaussian channels. This is exploited in the next two corollaries.

Corollary 4. Passive-environment bosonic channels $\mathcal{A}_{\mathrm{G}}^{\downarrow}$ are Fock-majorization preserving, that is, for all states $\rho$ and $\sigma$,

$$
\text { if } \rho \succ_{\mathrm{F}} \sigma \text {, then } \mathcal{A}_{\mathrm{G}}^{\downarrow}[\rho] \succ_{\mathrm{F}} \mathcal{A}_{\mathrm{G}}^{\downarrow}[\sigma] \text {. }
$$

Indeed, since $\mathcal{B}_{\eta}^{\downarrow}$ is passive-preserving (theorem 3), the duality property of passive-environment channels (corollary 3) combined with theorem 4 implies that $\mathcal{A}_{\mathrm{G}}^{\downarrow}$ satisfies the ladder of Fock-majorization relations $\mathcal{A}_{\mathrm{G}}^{\downarrow}[|i\rangle\langle i|] \succ_{\mathrm{F}} \mathcal{A}_{\mathrm{G}}^{\downarrow}[|i+1\rangle\langle i+1|], \forall i \geqslant 0$, hence it is Fockmajorization preserving as a consequence of theorem 1 .

Corollary 5. Passive-environment bosonic channels $\mathcal{A}_{\mathrm{G}}^{\downarrow}$ are passive preserving, that is

$$
\text { if } \rho^{\downarrow} \text { is passive, then } \mathcal{A}_{\mathrm{G}}^{\downarrow}\left[\rho^{\downarrow}\right] \text { is also passive. }
$$

Indeed, since $\mathcal{B}_{\eta}^{\downarrow}$ satisfies the ladder of Fock-majorization relations (8) (theorem 2), the duality property of passive-environment channels (corollary 3) combined with theorem 4 implies that $\mathcal{A}_{\mathrm{G}}^{\downarrow}$ is passive-preserving.

Finally, using these two corollaries and the equivalence between majorization and Fockmajorization for the set of passive states, we obtain the following.

Corollary 6. Passive-environment channels $\mathcal{A}_{\mathrm{G}}^{\downarrow}$ are majorization-preserving over the set of passive states, that is, for any two passive states $\rho^{\downarrow}$ and $\sigma^{\downarrow}$,

$$
\text { if } \rho^{\downarrow} \succ \sigma^{\downarrow} \text {, then } \mathcal{A}_{\mathrm{G}}^{\downarrow}\left[\rho^{\downarrow}\right] \succ \mathcal{A}_{\mathrm{G}}^{\downarrow}\left[\sigma^{\downarrow}\right] \text {. }
$$

\section{Conclusion}

In summary, we have shown that all bosonic quantum channels whose Stinespring dilation involves a Gaussian unitary (either a beam splitter or a two-mode squeezer) and a passive 
environment (from which no energy can be extracted by acting with a unitary) exhibit a series of properties regarding how the order or disorder (measured via majorization) is transfered across the channel. Our central result is that any such channel preserves the Fock-majorization relation (see corollary 1 for channel $\mathcal{B}_{\eta}^{\downarrow}$ and corollary 4 for $\mathcal{A}_{\mathrm{G}}^{\downarrow}$ ). Moreover, as a consequence of being passive-preserving, all these passive-environment channels equally preserve the regular majorization relation over the set of passive states (see corollary 2 for $\mathcal{B}_{\eta}^{\downarrow}$ and corollary 6 for $\mathcal{A}_{\mathrm{G}}^{\downarrow}$.

These results heavily rely on the Fock-majorization relation for bosonic systems. Because of its connection with energy, Fock-majorization can be viewed as the fundamental mathematical relation that is conserved when quantum states evolve through passive-environment channels, which allows one to relate the concepts of disorder and energy (when dealing with passive states, the concepts of majorization and Fock-majorization become equivalent). Our paper can thus be read in the context of quantum thermodynamics, where we define the class of passive-environment operations that encompass-but go beyond-thermal operations and characterize the properties of such operations in terms of majorization theory. These results will hopefully contribute to connect the area of continuous-variable bosonic channels together with quantum thermodynamics.

More generally, the notions of passive-environment channel and Fock-majorization relation are independent of the specific nature of the considered system, so we anticipate that our results can be extended to other quantum systems (beyond a bosonic mode) and arbitrary Hamiltonians (beyond the Harmonic oscillator). The energy-majorization relation between two states (based on comparing the diagonal elements in the energy eigenbasis) should then be conserved along the thermodynamical operation resulting from the energy-conserving coupling of the system with a passive environment (i.e. an environment state having the minimum energy compatible with its eigenspectrum).

\section{Acknowledgments}

We thank Ognyan Oreshkov for useful discussions. This work was supported by the FRS-FNRS under Project No. T.0224.18. MGJ also acknowledges support from the FRIA foundation.

\section{Appendix A. Extension of Fock-majorization preservation to non-diagonal states in the case of passive channels}

Here, we prove that theorem 1 can be applied to channels $\Phi \equiv \mathcal{B}_{\eta}^{\downarrow}$. We do this by showing that if $\rho$ is diagonal in the Fock basis, $\mathcal{B}_{\eta}^{\downarrow}[\rho]$ is also diagonal in the Fock basis, while if $\rho$ is non-diagonal in the Fock basis, its non-diagonal elements do not contribute to the diagonal elements of $\mathcal{B}_{\eta}^{\downarrow}[\rho]$. It can be shown that [16]

$$
\tilde{U}_{\theta}^{\mathrm{BS}}|i, k\rangle=\sum_{n=0}^{i+k} b_{n}^{(i, k)}(\theta)|n, i+k-n\rangle
$$

where

$$
b_{n}^{(i, k)}(\theta)=\sum_{m=\max (0, n-k)}^{\min (i, n)}(-1)^{i-m} \sqrt{\Gamma_{m, n-m}^{(i, k)}}(\cos \theta)^{2 m+k-n}(\sin \theta)^{i-2 m+n},
$$

and 


$$
\Gamma_{n, m}^{(i, k)}=\left(\begin{array}{c}
i \\
n
\end{array}\right)\left(\begin{array}{c}
k \\
m
\end{array}\right)\left(\begin{array}{c}
n+m \\
n
\end{array}\right)\left(\begin{array}{c}
i-n+k-m \\
i-n
\end{array}\right)
$$

If we define our passive channel as in equation (4) and set $\eta=\cos ^{2} \theta$, we have

$$
\begin{aligned}
\mathcal{B}_{\eta}^{\downarrow}[|i\rangle\langle j|]= & \sum_{k} \lambda_{k}^{\downarrow} \operatorname{Tr}_{\mathrm{E}}\left[\tilde{U}_{\theta}^{\mathrm{BS}}(|i\rangle\langle j|\otimes| k\rangle\langle k|) \tilde{U}_{\theta}^{\mathrm{BS} \dagger}\right] \\
=\sum_{k} \lambda_{k}^{\downarrow} \sum_{l}\left\langle\left. l\right|_{\mathrm{E}}\right. & \left(\sum_{n=0}^{i+k} b_{n}^{(i, k)}(\theta)|n, i+k-n\rangle\right) \\
& \times\left(\sum_{m=0}^{j+k}\left(b_{m}^{(j, k)}(\theta)\right)^{*}\langle m, j+k-m|\right)|l\rangle_{\mathrm{E}}
\end{aligned}
$$

so that

$$
\mathcal{B}_{\eta}^{\downarrow}[|i\rangle\langle j|]=\sum_{k} \lambda_{k}^{\downarrow} \sum_{n} b_{n}^{(i, k)}(\theta)\left(b_{n+j-i}^{(j, k)}(\theta)\right)^{*}|n\rangle\langle n+j-i| .
$$

When the input state is diagonal, we end up with

$$
\mathcal{B}_{\eta}^{\downarrow}[|i\rangle\langle i|]=\sum_{k} \lambda_{k}^{\downarrow} \sum_{n}\left|b_{n}^{(i, k)}(\theta)\right|^{2}|n\rangle\langle n|, \quad \eta=\cos ^{2} \theta,
$$

which means that if $\rho$ is diagonal in the Fock basis, $\mathcal{B}_{\eta}^{\downarrow}[\rho]$ is also diagonal in the Fock basis. Furthermore, equation (A.4) tells us that if $\rho$ is non-diagonal in the Fock basis, its non-diagonal elements do not contribute to the diagonal elements of $\mathcal{B}_{\eta}^{\downarrow}[\rho]$. This analysis can be trivially extended to channels $\Phi \equiv \mathcal{A}_{\mathrm{G}}^{\downarrow}$.

\section{Appendix B. Unitary reversal in channels with a Fock-diagonal state of the environment}

Consider a channel

$$
\tilde{\mathcal{C}}_{\theta}^{\mathrm{BS}}[\bullet]=\operatorname{Tr}_{\mathrm{E}}\left[\tilde{U}_{\theta}^{\mathrm{BS}}\left(\bullet \otimes \sigma_{\mathrm{E}}\right) \tilde{U}_{\theta}^{\mathrm{BS} \dagger}\right]
$$

where the state of the environment $\sigma_{\mathrm{E}}=\sum_{k} \sigma_{k}|k\rangle\langle k|$ is diagonal in the Fock basis. We show here that in this case, $\tilde{\mathcal{C}}_{\theta}^{\mathrm{BS}}=\tilde{\mathcal{C}}_{-\theta}^{\mathrm{BS}}$. From equation (A.4), we have

$$
\tilde{\mathcal{C}}_{\theta}^{\mathrm{BS}}[|i\rangle\langle j|]=\sum_{k} \sigma_{k} \sum_{n} c_{n, j}^{(i, k)}(\theta)|n\rangle\langle n+j-i|,
$$

with

$$
\tilde{b}_{n, j}^{(i, k)}(\theta)=b_{n}^{(i, k)}(\theta)\left(b_{n+j-i}^{(j, k)}(\theta)\right)^{*}
$$

so that

$$
\begin{array}{r}
\tilde{b}_{n, j}^{(i, k)}(\theta)=\sum_{m_{1}=\max (0, n-k)}^{\min (i, n)} \sum_{m_{2}=\max (0, n+j-i-k)}^{j+\min (0, n-i)}(-1)^{i+j-m_{1}-m_{2}} \sqrt{\Gamma_{m_{1}, n-m_{1}}^{(i, k)} \Gamma_{m_{2}, n+j-i-m_{2}}^{(j, k)}} \\
\times(\cos \theta)^{2\left(m_{1}+m_{2}+k-n\right)-j+i}(\sin \theta)^{2\left(n+j-m_{1}-m_{2}\right)},
\end{array}
$$


which trivially implies that

$$
\tilde{b}_{n, j}^{(i, k)}(-\theta)=\tilde{b}_{n, j}^{(i, k)}(\theta),
$$

and

$$
\tilde{\mathcal{C}}_{-\theta}^{\mathrm{BS}}[\bullet]=\tilde{\mathcal{C}}_{\theta}^{\mathrm{BS}}[\bullet],
$$

for any input state. Consider now a channel

$$
\tilde{\mathcal{C}}_{r}^{\mathrm{TMS}}[\bullet]=\operatorname{Tr}_{\mathrm{E}}\left[\tilde{U}_{r}^{\mathrm{TMS}}\left(\bullet \otimes \sigma_{\mathrm{E}}\right) \tilde{U}_{r}^{\mathrm{TMS} \dagger}\right]
$$

where, again, the state of the environment $\sigma_{\mathrm{E}}=\sum_{k} \sigma_{k}|k\rangle\langle k|$ is diagonal in the Fock basis. If we define

$$
a_{n}^{(i, k)}(r)=\left\langle n, m\left|\tilde{U}_{r}^{\mathrm{TMS}}\right| i, k\right\rangle, \quad m=n-i+k,
$$

we have

$$
\tilde{\mathcal{C}}_{r}^{\mathrm{TMS}}[|i\rangle\langle j|]=\sum_{k} \sigma_{k} \sum_{m} \tilde{a}_{m+i-k}^{(i, k)}(r)|m+i-k\rangle\langle m+j-k|
$$

where

$$
\tilde{a}_{m+i-k, j}^{(i, k)}(r)=a_{m+i-k}^{(i, k)}(r)\left(a_{m+j-k}^{(j, k)}(r)\right)^{*} .
$$

The partial time reversal property of equation (32) implies that

$$
a_{m}^{(i, k)}(r)=(\cos \theta) b_{m}^{(i, m+k-i)}(\theta),
$$

where $\theta$ and $r$ verify $\cos \theta=\operatorname{sech} r$, so that

$$
\tilde{a}_{m+i-k, j}^{(i, k)}(r)=(\cos \theta)^{2} b_{m+i-k}^{(i, m)}(\theta)\left(b_{m+j-k}^{(j, m)}(\theta)\right)^{*}=(\cos \theta)^{2} \tilde{b}_{m+i-k, j}^{(i, m)}(\theta),
$$

which leads to

$$
\tilde{a}_{m+i-k, j}^{(i, k)}(-r)=\tilde{a}_{m+i-k, j}^{(i, k)}(r),
$$

and

$$
\tilde{\mathcal{C}}_{-r}^{\mathrm{TMS}}[\bullet]=\tilde{\mathcal{C}}_{r}^{\mathrm{TMS}}[\bullet],
$$

for any input state.

\section{ORCID iDs}

Michael G Jabbour (10 https://orcid.org/0000-0003-1851-9540

\section{References}

[1] Åberg J 2013 Nat. Commun. 41925

[2] Horodecki M and Oppenheim J 2013 Nat. Commun. 42059

[3] Skrzypczyk P, Short A J and Popescu S 2014 Nat. Commun. 54185

[4] Gour G, Müller M P, Narasimhachar V, Spekkens R W and Halpern N Y 2015 Phys. Rep. 5831

[5] Brandão F, Horodecki M, Ng N, Oppenheim J and Wehner S 2015 Proc. Natl Acad. Sci. 1123275 
[6] Marshall A W and Olkin I 1979 Inequalities: Theory of Majorization and its Applications (New York: Academic)

[7] Nielsen M A 1999 Phys. Rev. Lett. 83436

[8] Jabbour M G, García-Patrón R and Cerf N J 2015 Phys. Rev. A 91012316

[9] García-Patrón R, Navarrete-Benlloch C, Lloyd S, Shapiro J H and Cerf N J 2012 Phys. Rev. Lett. 108110505

[10] Gagatsos C N, Oreshkov O and Cerf N J 2013 Phys. Rev. A 87042307

[11] Mari A, Giovannetti V and Holevo A S 2014 Nat. Commun. 53826

[12] Giovannetti V, Holevo A S and Mari A 2015 Theor. Math. Phys. 182284

[13] De Palma G, Trevisan D and Giovannetti V 2016 IEEE Trans. Inf. Theory 622895

[14] Sabapathy K K and Winter A 2017 Phys. Rev. A 95062309

[15] Jabbour M G, García-Patrón R and Cerf N J 2016 New J. Phys. 18073047

[16] Jabbour M G and Cerf N J 2018 (arXiv:1803.10734 [quant-ph])

[17] Pusz W and Woronowicz S L 1978 Commun. Math. Phys. 58273

[18] Lenard A 1978 J. Stat. Phys. 19575

[19] Wehrl A 1978 Rev. Mod. Phys. 5022

[20] Goold J, Huber M, Riera A, del Rio L and Skrzypczyk P 2016 J. Phys. A: Math. Theor. 49143001

[21] Horodecki M, Horodecki P and Oppenheim J 2003 Phys. Rev. A 67062104

[22] Weedbrook C, Pirandola S, García-Patrón R, Ralph T, Cerf N J, Shapiro J H and Lloyd S 2012 Rev. Mod. Phys. 84621

[23] Narasimhachar V and Gour G 2015 Nat. Commun. 67689

[24] Jabbour M G and Cerf N J (in preparation) 